\title{
Cost-Effectiveness of Thrombolysis within 4.5 Hours of Acute Ischemic Stroke in China
}

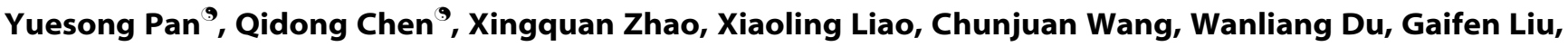 Liping Liu, Chunxue Wang, Yilong Wang*, Yongjun Wang', for the TIMS-CHINA investigators"}

Department of Neurology, Beijing Tiantan Hospital, Capital Medical University, Beijing, China

\begin{abstract}
Background: Previous economic studies conducted in developed countries showed intravenous tissue-type plasminogen activator (tPA) is cost-effective for acute ischemic stroke. The present study aimed to determine the cost-effectiveness of tPA treatment in China, the largest developing country.

Methods: A combination of decision tree and Markov model was developed to determine the cost-effectiveness of tPA treatment versus non-tPA treatment within 4.5 hours after stroke onset. Outcomes and costs data were derived from the database of Thrombolysis Implementation and Monitor of acute ischemic Stroke in China (TIMS-China) study. Efficacy data were derived from a pooled analysis of ECASS, ATLANTIS, NINDS, and EPITHET trials. Costs and quality-adjusted life-years (QALYs) were compared in both short term (2 years) and long term (30 years). One-way and probabilistic sensitivity analyses were performed to test the robustness of the results.

Results: Comparing to non-tPA treatment, tPA treatment within 4.5 hours led to a short-term gain of 0.101 QALYs at an additional cost of CNY 9,520 (US\$ 1,460), yielding an incremental cost-effectiveness ratio (ICER) of CNY 94,300 (US\$ 14,500) per QALY gained in 2 years; and to a long-term gain of 0.422 QALYs at an additional cost of CNY 6,530 (US\$ 1,000), yielding an ICER of CNY 15,500 (US\$ 2,380) per QALY gained in 30 years. Probabilistic sensitivity analysis showed that tPA treatment is cost-effective in $98.7 \%$ of the simulations at a willingness-to-pay threshold of CNY 105,000 (US\$16,200) per QALY.
\end{abstract}

Conclusions: Intravenous tPA treatment within 4.5 hours is highly cost-effective for acute ischemic strokes in China.

Citation: Pan Y, Chen Q, Zhao X, Liao X, Wang C, et al. (2014) Cost-Effectiveness of Thrombolysis within 4.5 Hours of Acute Ischemic Stroke in China. PLoS ONE 9(10): e110525. doi:10.1371/journal.pone.0110525

Editor: Maarten Postma, Groningen Research Institute of Pharmacy, Netherlands

Received January 23, 2014; Accepted September 22, 2014; Published October 20, 2014

Copyright: $(\subset) 2014$ Pan et al. This is an open-access article distributed under the terms of the Creative Commons Attribution License, which permits unrestricted use, distribution, and reproduction in any medium, provided the original author and source are credited.

Funding: This study was funded by the State Key Development Program of Basic Research of China (2009CB521905), and National Science and Technology Majo Project of China (2011BAI08B02). The funders had no role in study design, data collection and analysis, decision to publish, or preparation of the manuscript.

Competing Interests: The authors have declared that no competing interests exist.

*Email: yongjunwang1962@gmail.com; yilong528@gmail.com

9 These authors contributed equally to this work.

- Investigators for the TIMS-CHINA project are listed in the Acknowledgments.

\section{Introduction}

Stroke accounts for 301 million disability-adjusted life-years, making it the first leading cause of death and imposing significant disease and economic burden in China [1]. Randomized controlled trials and large observational studies have demonstrated the effectiveness with acceptable safety of intravenous recombinant tissue-type plasminogen activator (tPA) for patients within 4.5 hours after onset of acute ischemic stroke [2-6].

Economic studies conducted in North America, Europe and Australia showed tPA given within 4.5 hours is cost-effective or even cost-saving in the long term [7-15]. However, all of these studies were conducted in developed countries; the findings from these countries may not be relevant in developing countries due to their differences in demographics, healthcare systems and payment coverage. Compared with developed countries, the low- and middle- income countries suffer higher mortality burden of stroke [16], and have lower percentage of patients with ischemic stroke treated with tPA [17]. On the other hand, most developed countries, such as United States, implement prospective payment system based on diagnosis related groups $[18,19]$. While in China, the payments are based on each clinical service. Particularly, the drug costs account for a large part of total costs for Chinese stroke patients. They pay CNY 8,197 (US\$ 1,261) just for $70 \mathrm{mg}$ tPA alone [20]. Is the expensive tPA still cost-effective and should it be widely generalized in developing countries like China? Economic analysis of tPA in developing countries is urgent.

Little is known about verifying the cost-effectiveness of tPA treatment in China. We sought to determine the cost-effectiveness of tPA within 4.5 hours after onset of acute ischemic stroke, using the data from the Thrombolysis Implementation and Monitor of acute ischemic Stroke in China (TIMS-China), which is a nationwide prospective registry of thrombolytic therapy with intravenous tPA in patients with acute ischemic stroke between May 2007 and April 2012. TIMS-China has recruited 1,440 consecutive tPA treated patients from 67 centers [21]. 


\section{Methods}

\section{Model Overview}

We adhered to the recommendations of the Panel on Costeffectiveness in Health and Medicine [22], including (1) components belonging in the numerator and denominator of a costeffectiveness (C/E) ratio; (2) measuring terms in the numerator of a $\mathrm{C} / \mathrm{E}$ ratio; (3) valuing health consequences in the denominator of a C/E ratio; (4) estimating effectiveness of interventions; (5) incorporating time preference and discounting; and (6) handling uncertainty. A combination of decision tree and Markov model (Figure 1) was developed to simulate the long-term (30 years) costeffectiveness of tPA treatment versus absence of tPA treatment within 4.5 hours after the onset of stroke. Our study was based on data from 1128 patients with acute ischemic stroke who received intravenous tPA within 4.5 hours in TIMS-CHINA [21]. The base case of model was a cohort of 100,000 patients (39\% female), with mean age of 63 years old, arriving at hospital within 4.5 hours after onset of stroke, whose clinical and demographic characteristics are same as patients enrolled in TIMS-CHINA study (Table 1). Total costs and quality-adjusted life-years (QALYs) gained with each alternative were estimated for each health state at 90 days from the index events and then estimated annually for the remainder 30 years. This analysis was conducted from the perspective of healthcare payers, including the government, medical insurance and patients.

\section{Input Parameters}

The baselines of patients and their outcomes in the three time windows (0-1.5, 1.5-3, 3-4.5 hours after the onset of stroke) of the tPA group, were obtained from the observed data directly drawn from the TIMS-CHINA study database (Table 2). Odds ratios of the favorable functional outcome (modified Rankin Scale (mRS) $0-1)$, death and symptomatic intracerebral hemorrhage $(\mathrm{sICH})$ in each time window were derived from a pooled analysis of the European Cooperative Acute Stroke Study Trial (ECASS), the Alteplase Thrombolysis for Acute Noninterventional Therapy in Ischemic Stroke Trial (ATLANTIS), the National Institute of Neurological Disorders and Stroke (NINDS), and the Echoplanar Imaging Thrombolytic Evaluation Trial (EPITHET) [23]. Odds ratio of sICH within $0-1.5$ hours were assumed to be the same as that of within 1.5-3 hours. Favorable functional outcome, death and $\mathrm{sICH}$ rates in the non-tPA treatment group were obtained from the observed data of the tPA group and the odds ratios of previous study [23]. The proportional distribution of patients remaining in categories of mRS 2-3 and mRS 4-5 at 90 days in the control group was assumed to be the same as that in tPA group.

Recurrent rates of stroke and mortality rates of recurrent strokes in years after the first 90 days were estimated from the China National Stroke Registry (CNSR, a nationwide registry for patients with acute cerebrovascular events in China between September 2007 and August 2008, recruiting 21,902 consecutive patients from 132 hospitals in China) [24]. We further assumed an increase in stroke recurrence rates by 1.019-fold per life year, according to the relative risk estimated from patients of ischemic stroke in GNSR.

Age specific non-stroke mortality rates were derived from the most recent published census of China and adjusted by the causes of death of 2010 reported in the China Health Statistics Yearbook 2012 [25,26]. Disability status were assumed to affect survival rate, therefore the final age specific non-stroke death rates in the model were adjusted according to the $\mathrm{mRS}$-specific death hazard ratios $[7,27]$. Patients remaining alive after stroke recurrence were assumed to be reallocated equally among categories of equal and greater disability [12]. For example, patients with minor or moderate disability who had a recurrent stroke and survived were allocated equally among minor or moderate disability category and severe disability category.

\section{Costs}

The total costs including both out-of-pocket costs and reimbursements, were converted to 2011 Chinese Yuan Renminbi (CNY) using the medical care component of consumer price index [26]. The average cost of single hospitalization after stroke was obtained from CNSR and the China Health Statistics Yearbook 2012 [26]. Annual post-hospitalization costs (such as inpatient and outpatient rehabilitation, ambulatory care and second prevention costs) were also obtained from GNSR. We estimated the additional costs of tPA treatment and occurrence of sICH after thrombolysis using the data from GNSR and TIMS-CHINA. Indirect economic costs such as lost work productivity were not included in this study. All costs and utilities were discounted by $3 \%$ per year [12].

Table 1. Clinical and Demographic Characteristics of Patients Treated with Tissue Plasminogen Activator within 4.5 Hours in TIMS-CHINA Study $(\mathrm{N}=1128)$.

\begin{tabular}{ll}
\hline Characteristic & \\
\hline Age (year), mean (SD) & $63.48(11.34)$ \\
Female, $\mathrm{n}(\%)$ & $440(39.01)$ \\
\hline Preadmission modified Rankin Score 0-1, $\mathrm{n}(\%)$ & $1081(95.83)$ \\
NIHSS at admission, median(Q1-Q3) & $11(7-16)$ \\
Hypertension, $\mathrm{n}(\%)$ & $667(59.13)$ \\
Diabetes mellitus, $\mathrm{n}(\%)$ & $196(17.38)$ \\
Dyslipidemia, $\mathrm{n}(\%)$ & $73(6.47)$ \\
Atrial fibrillation, $\mathrm{n}(\%)$ & $202(17.91)$ \\
Current smoking, $\mathrm{n}(\%)$ & $387(34.31)$ \\
History of stroke, $\mathrm{n}(\%)$ & $208(18.44)$ \\
\hline
\end{tabular}

TIMS-CHINA, Thrombolysis Implementation and Monitor of acute ischemic Stroke in China.

doi:10.1371/journal.pone.0110525.t001 


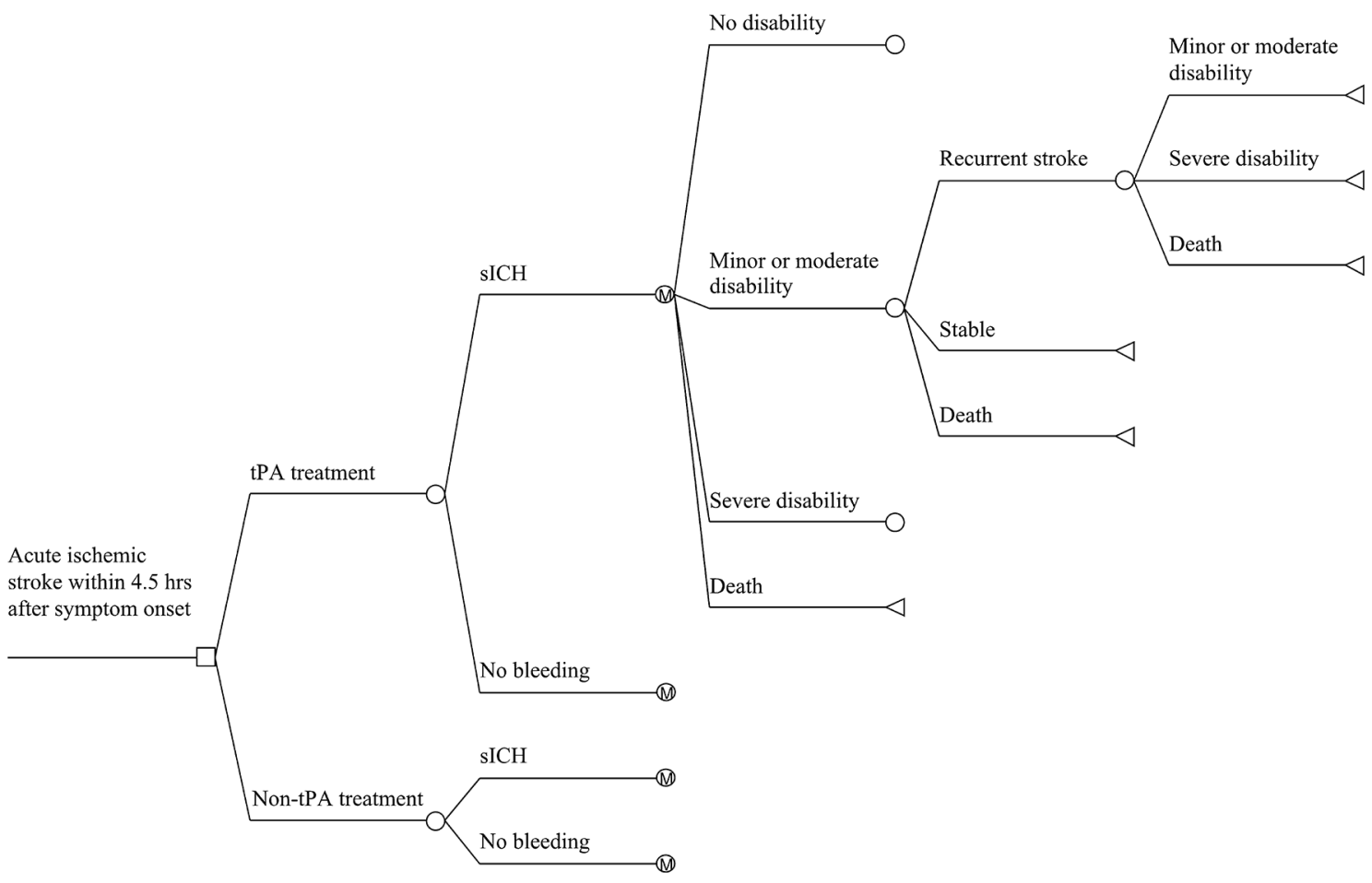

Figure 1. Markov state-transition model. All modeled patients started at 63 years old with an acute ischemic stroke receiving either intravenous tissue-type plasminogen activator (tPA) or no tPA treatment within 4.5 hours after the onset of stroke, and transition between health states was tracked until death or the 30-year time horizon is reached. Patients remaining alive after recurrent stroke may transit to category of equal or greater disability. Only transition from minor or moderate disability category was illustrated in the figure. $\mathrm{M}=$ Markov node; $s \mathrm{CH}=\mathrm{symptomatic}$ intracerebral hemorrhage.

doi:10.1371/journal.pone.0110525.g001

\section{Health States}

Patients could undergo transitions between four disability states according to functional outcome based on mRS: no disability (mRS 0-1), minor or moderate disability (mRS 2-3), severe disability (mRS 4-5) and dead (mRS 6) [28]. At the end of each cycle, patients could remain in their current health state, transit to a lower level health state due to recurrent stroke, or die due to a recurrent stroke or a non-stroke cause (see Figure 1).

Table 2. Outcomes and Efficacy of Patients Treated with tPA by Different Time From Onset to Needle.

\begin{tabular}{|c|c|c|c|c|}
\hline Model Input & $0-1.5 \mathrm{hrs}$ & $1.5-3 \mathrm{hrs}$ & 3-4.5 hrs & Reference \\
\hline Percentage of patients & 5.68 & 61.13 & 33.19 & TIMS-CHINA database \\
\hline \multicolumn{5}{|c|}{ Functional outcome at 90 days after tPA treatment (\%): } \\
\hline No disability (mRS $0-1$ ) & 53.97 & 47.11 & 48.09 & \\
\hline Minor or moderate disability (mRS 2-3) & 23.81 & 24.89 & 25.14 & \\
\hline Severe disability (mRS 4-5) & 9.52 & 18.37 & 15.30 & \\
\hline Death (mRS 6) & 12.70 & 9.63 & 11.47 & \\
\hline sICH (per ECASS II definition) & 4.69 & 3.05 & 5.35 & \\
\hline \multicolumn{5}{|l|}{ Odds ratio $(95 \% \mathrm{Cl})$ at day 90} \\
\hline \multirow[t]{2}{*}{ mRS 0-1 } & 2.55 & 1.64 & 1.34 & 23 \\
\hline & $(1.44-4.52)$ & $(1.12-2.40)$ & $(1.06-1.68)$ & \\
\hline \multirow[t]{2}{*}{ Death } & 0.78 & 1.13 & 1.22 & 23 \\
\hline & $(0.41-1.48)$ & $(0.70-1.82)$ & $(0.87-1.71)$ & \\
\hline \multirow[t]{2}{*}{ sICH } & 8.23 & 8.23 & 3.61 & 23 \\
\hline & $(2.39-28.32)$ & $(2.39-28.32)$ & $(1.76-7.38)$ & \\
\hline
\end{tabular}

tPA, tissue plasminogen activator; mRS, modified Rankin Score; sICH, symptomatic intracerebral hemorrhage; ECASS II, Second European-Australasian Acute Stroke Study; TIMS-CHINA, Thrombolysis Implementation and Monitor of acute ischemic Stroke in China.

doi:10.1371/journal.pone.0110525.t002 


\section{Outcome Assessment}

Health outcomes were measured in QALYs by multiplying years of life by utility scores derived from the literature. Utility estimates were based on published utility values stratified by mRS category $[12,29,30]$. Economic outcomes were measured in cumulative direct medical costs associated with stroke. The incremental cost-effectiveness ratio (ICER) was calculated by dividing the incremental costs by the incremental QALYs. We modeled outcomes and costs over the short-term (2 years) and the long-term (30 years). Using the willingness-to-pay threshold recommended by the Commission on Macroeconomics and Health of World Health Organization [31], the intervention was considered cost-effective if the ICER was less than GNY 105,000 (3x GDP per capita of China in 2011 [26], US\$ 16,200) per QALY gained.

\section{Sensitivity Analysis}

The robustness of the model was tested by means of one-way sensitivity analyses for all variables across plausible ranges, which were obtained from the literature (Table 2, Table 3). To evaluate the impact of the parameter and stochastic uncertainty in all variables varied simultaneously, a probabilistic sensitivity analysis was performed using Monte Carlo simulation in Ersatz v1.3 (a bootstrap add-in for Microsoft Excel for Windows; EpiGear International Pty Ltd, Brisbane, Australia). We assumed the probabilities and utilities followed a beta distribution, and costs followed a lognormal distribution. The simulation was run 10,000 times to capture stability of the results. A scatter-plot and a costeffectiveness acceptability curve were developed to represent uncertainty. Sensitivity analyses were only applied to the long-term (30 years) results.

\section{Results}

\section{Base Case Analysis}

Table 4 shows the outcomes, costs and ICER calculated in the short term (1, 2 years) and in the long term (30 years). In the basecase scenario, for a 63-year-old patient with acute ischemic stroke, tPA treatment would be cost-ineffective in the first year, but become cost-effective from the second year onwards, using the threshold of CNY 105,000 (3x GDP per capita of China in 2011, US\$ 16,200) as the willingness-to-pay per QALY. After 2 years, tPA treatment gained 0.101 QALYs at an additional cost of CNY 9,520 (US\$ 1,460), yielding an ICER of CNY 94,300 (US\$ 14,500) per QALY gained. In the long term (30 years), tPA treatment gained 0.422 QALYs at an additional cost of CNY 6,530 (US\$ 1,000), yielding an ICER of CNY 15,500 (US\$ 2,380) per QALY gained.

\section{Sensitivity Analysis}

One-way sensitivity analysis showed the results in the long term are robust. Tornado diagrams illustrated the effect of varying input parameters on the long term ICER (Figure 2). Overall, the ICER was most sensitive to odds ratio of favorable functional outcome at day 90 within 1.5-3 hours and annual post-hospitalization costs (mRS 2-5). If odds ratio of favorable functional outcome at day 90 within 1.5-3 hours increased to 2.40 from 1.12, the ICER of tPA treatment would drop to CNY 9.653/QALY from CNY 40,667/ QALY. If annual post-hospitalization cost of disabling stroke (mRS 2-5) increased from CNY 2,592 to GNY 12,959, the ICER would decrease from CNY 37,014/QALY to GNY 8,057/QALY. In contrast, the ICER was relatively insensitive to odds ratio of sICH at day 90 and one-time hospitalization costs.
Results of the probabilistic sensitivity analysis in the long term are shown in Figure 3. Among the 10,000 simulation runs, there was a $14.4 \%$ chance that tPA treatment turned to be less costly and more effective than non-tPA treatment. tPA treatment was cost-effective in $98.7 \%$ of the simulations at a willingness-to-pay threshold of CNY 105,000 (3x GDP per capita of China in 2011, US\$ 16,200) per QALY, and still remained cost-effective in $79.2 \%$ of the simulations at a threshold of CNY 35,100 (1x GDP per capita of China in 2011, US\$ 5,400) per QALY. The costeffectiveness acceptability curve of tPA treatment is shown in Figure 4.

\section{Discussion}

Our study indicated that tPA treatment for acute ischemic stroke within 4.5 hours was cost-effective not only in the short term (2 years), but also highly cost-effective in the long term (30 years) in China. A patient with acute ischemic stroke treated with tPA within 4.5 hours gain an ICER of CNY 15,500 per QALY, which was below 1x GDP per capita of China in 2011 (CNY 105,000). By contrast, cost effectiveness analyses conducted in some developed countries demonstrated tPA treatment was cost-saving in the long term [7-10]. We also found that the ICER is less sensitive to the additional costs of $\mathrm{tPA}$ treatment, but more sensitive to odds ratio of favorable functional outcome at day 90 within 1.5-3 hours and annual post-hospitalization costs (Figure 2). The reason might be that $\mathrm{PA}$ treatment is highly effective in functional outcome for acute ischemic stroke $(\mathrm{OR}=1.64$ for 1.5-3 hours [23]), and also dramatically influence the long-term utility and costs, which may offset the costs of tPA treatment.

The differences between China and the developed countries may influence the cost-effectiveness of tPA treatment. First, unlike the diagnosis related payment system in most developed countries $[18,19]$, China implements payment system according to the clinical services. The stroke-care related payments in China included medical care covered by government, medical insurance for urban workers, rural cooperative medical care, commercial medical insurance, plus patient own expense, in the proportions of $7.2 \%, 53.6 \%, 16.9 \%, 0.7 \%$ and $21.6 \%$, respectively, according to CNSR (unpublished data). The out-of-pocket costs were only $10 \%$ to $20 \%$ of the total costs if the payment was covered by government. For urban workers with medical insurance, their average 3-month hospital and medication costs due to stroke were GNY 16,525 (US\$2,361), while the out-of-pocket costs were CNY 14,478 (US\$ 2,068) in 2006 [32]. However, for the patients without coverage from the government or medical insurance, they had to pay more out-of-pocket costs, with little support from state. No matter which payment resources that the stroke patients had, the total costs depended on the service and medicine prescribed, with huge variation in hospitals and geographical regions in China. In brief, Chinese patients must pay for all the costs first, and then search for corresponding reimbursement after the therapy process finished. For the patients without tPA treatment, their one-time hospitalization cost was CNY 9,526 if they had mRS 0-1, while if they had tPA treatment, the cost due to tPA alone was CNY 8,197 (US\$ 1,261) in China [20]. Therefore, the cost of tPA in China were relatively higher than that in developed countries. Second, several surveys showed the lack of organized rehabilitation and decreased adherence to secondary prevention of ischemic stroke in China after discharge [33-36]. Therefore, the long-term post-hospitalization care costs might be similar between the tPA treatment group and the control group in China. Due to the facts above, clinically, it is very important to estimate the cost- 
Table 3. Base-case and the Range of Values Used in the Model.

\begin{tabular}{|c|c|c|c|}
\hline Model Input & Base Case & Range & Reference \\
\hline \multicolumn{4}{|l|}{ Probabilities inputs } \\
\hline Recurrent rate of stroke (per patient year) & 0.1181 & $0.1123-0.1241$ & CNSR \\
\hline Relative risk of stroke recurrence per life year & 1.019 & $1.014-1.024$ & CNSR \\
\hline Death rate with recurrent stroke & 0.2101 & $0.1887-0.2316$ & CNSR \\
\hline Age specific non-stroke death rate* & $0.0089-0.1654$ & & 25,26 \\
\hline Non-stroke death hazard ratios & & & 27 \\
\hline mRS 0-1 & 1 & $1.0-1.2$ & \\
\hline mRS 2-3 & 1.19 & $1.1-1.3$ & \\
\hline $\mathrm{mRS} 4-5$ & 2.04 & $1.4-3.0$ & \\
\hline \multicolumn{4}{|l|}{ Cost inputs (2011 Chinese Yuan Renminbi) } \\
\hline Additional costs of tPA treatment & 10830 & $8385-12630$ & CNSR, TIMS-CHINA \\
\hline Additional costs of sICH & 2300 & $500-4800$ & TIMS-CHINA \\
\hline \multicolumn{4}{|l|}{ One-time hospitalization costs } \\
\hline mRS 0-1 & 9526 & 5502-11994 & CNSR, 26 \\
\hline mRS 2-5 & 12595 & 6922-16516 & \\
\hline mRS 6 & 10794 & $5072-14267$ & \\
\hline \multicolumn{4}{|l|}{ Annual post-hospitalization costs } \\
\hline mRS $0-1$ & 6773 & 2028-8639 & CNSR \\
\hline mRS 2-5 & 10305 & 2592-12959 & \\
\hline \multicolumn{4}{|l|}{ Utility inputs } \\
\hline No disability (mRS $0-1$ ) & 0.80 & $0.80-0.95$ & $12,29,30$ \\
\hline Minor or moderate disability (mRS $2-3$ ) & 0.58 & $0.56-0.78$ & \\
\hline Severe disability (mRS 4-5) & 0.28 & $0.05-0.36$ & \\
\hline Death (mRS 6) & 0.00 & $0.00-0.00$ & \\
\hline \multicolumn{4}{|l|}{ Discount rate inputs } \\
\hline Costs & 0.03 & $0.03-0.08$ & 12 \\
\hline Outcomes & 0.03 & $\pm 20 \%$ & 12 \\
\hline
\end{tabular}

All costs were converted to 2011 Chinese Yuan Renminbi by using the medical care component of consumer price index; to convert CNY to US dollars, divide by 6.5 . tPA, tissue plasminogen activator; mRS, modified Rankin Score; sICH, symptomatic intracerebral hemorrhage; CNSR, China National Stroke Registry. TIMS-CHINA, Thrombolysis Implementation and Monitor of acute ischemic Stroke in China.

*Age specific non-stroke death rate: only the number of 63 years old $(0.0089)$ and 93 years old $(0.1654)$ are presented.

doi:10.1371/journal.pone.0110525.t003

effectiveness in China. And these facts might partly explain the difference in ICER between China and developed countries.

This study has several limitations. First, the costs used in the model may not be accurate for each component of tPA related treatments, tPA drugs, extra MRI scanning, extra consultant and nursing services, change in length of stay, and so on [10].
However, we think the overall costs were recorded accurately in the total hospitalization cost. We estimated the additional costs of tPA treatment on the basis of the difference of the total hospitalization costs of sICH-free patients with tPA treatment in TIMS-CHINA study and the hospitalization costs of patients without tPA treatment in CNSR. We calculated the additional

Table 4. Costs and Outcomes per Capita in Base-case Analysis.

\begin{tabular}{lllll}
\hline \multicolumn{1}{l}{ Time horizon } & Treat strategy & QALYs & Cost (CNY) & ICER (CNY/QALY) \\
\hline 1 year & No tPA & 0.655 & 20,680 & - \\
& tPA & 0.711 & 30,850 & 181,607 \\
2 years & No tPA & 1.102 & 29,460 & - \\
& tPA & 1.203 & 38,980 & 94,257 \\
\hline 0 years & No tPA & 4.571 & 114,350 & - \\
& tPA & 4.993 & 120,880 & 15,474 \\
\hline
\end{tabular}

tPA, tissue plasminogen activator; QALY, quality-adjusted life-year; ICER, incremental cost-effectiveness ratio.

doi:10.1371/journal.pone.0110525.t004 


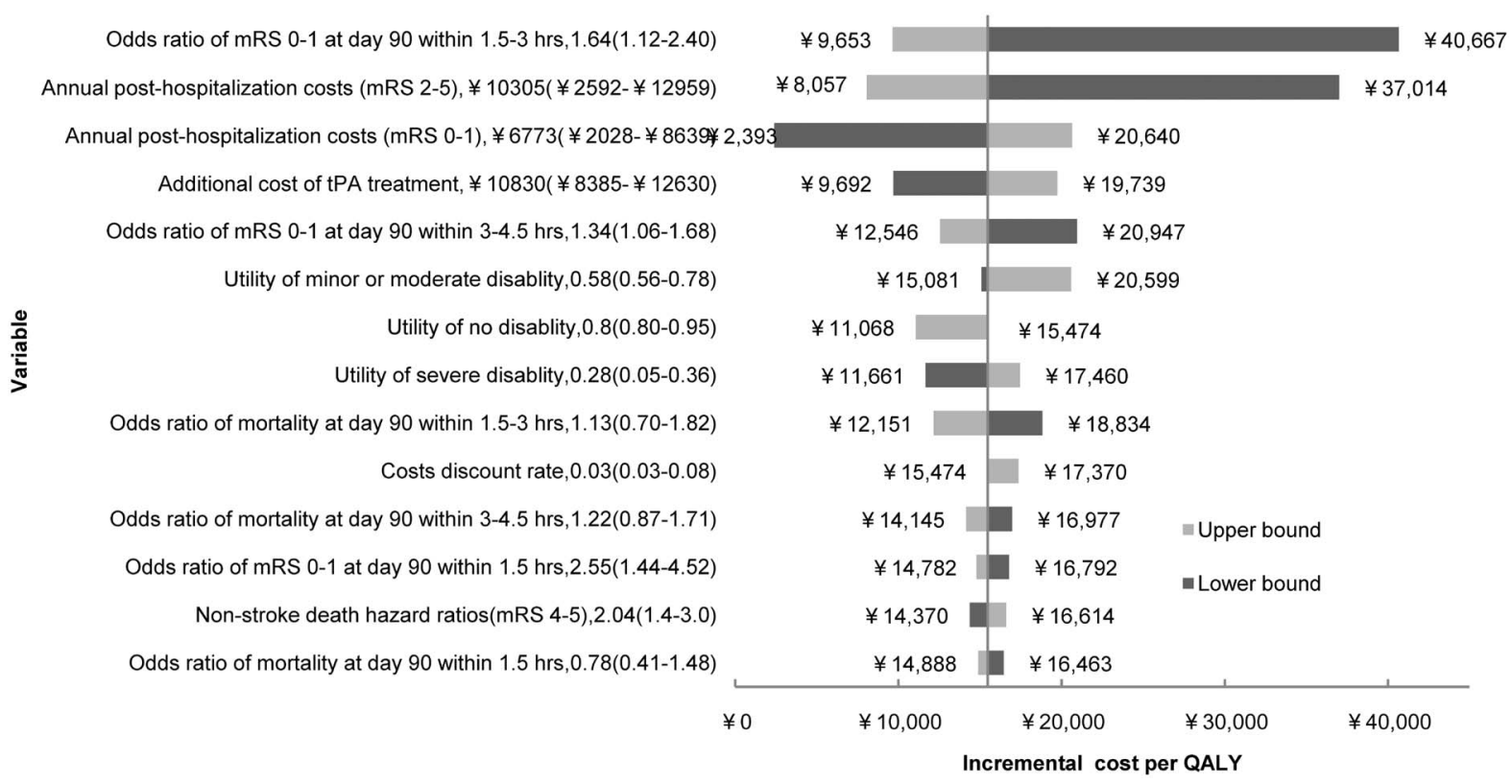

Figure 2. One-way sensitivity analyses on incremental cost-effectiveness ratio (ICER) gained in the long term ( 30 years) by tPA treatment within 4.5 hours. All model parameters were analyzed, and only those with the highest relative effect on ICER are displayed. Dark shaded bars represent the lower bound, while light shaded bars represent the upper bound of the parameter range. Base-case scenario of ICER is CNY 15,500 per quality-adjusted life-year (QALY) gained.

doi:10.1371/journal.pone.0110525.g002

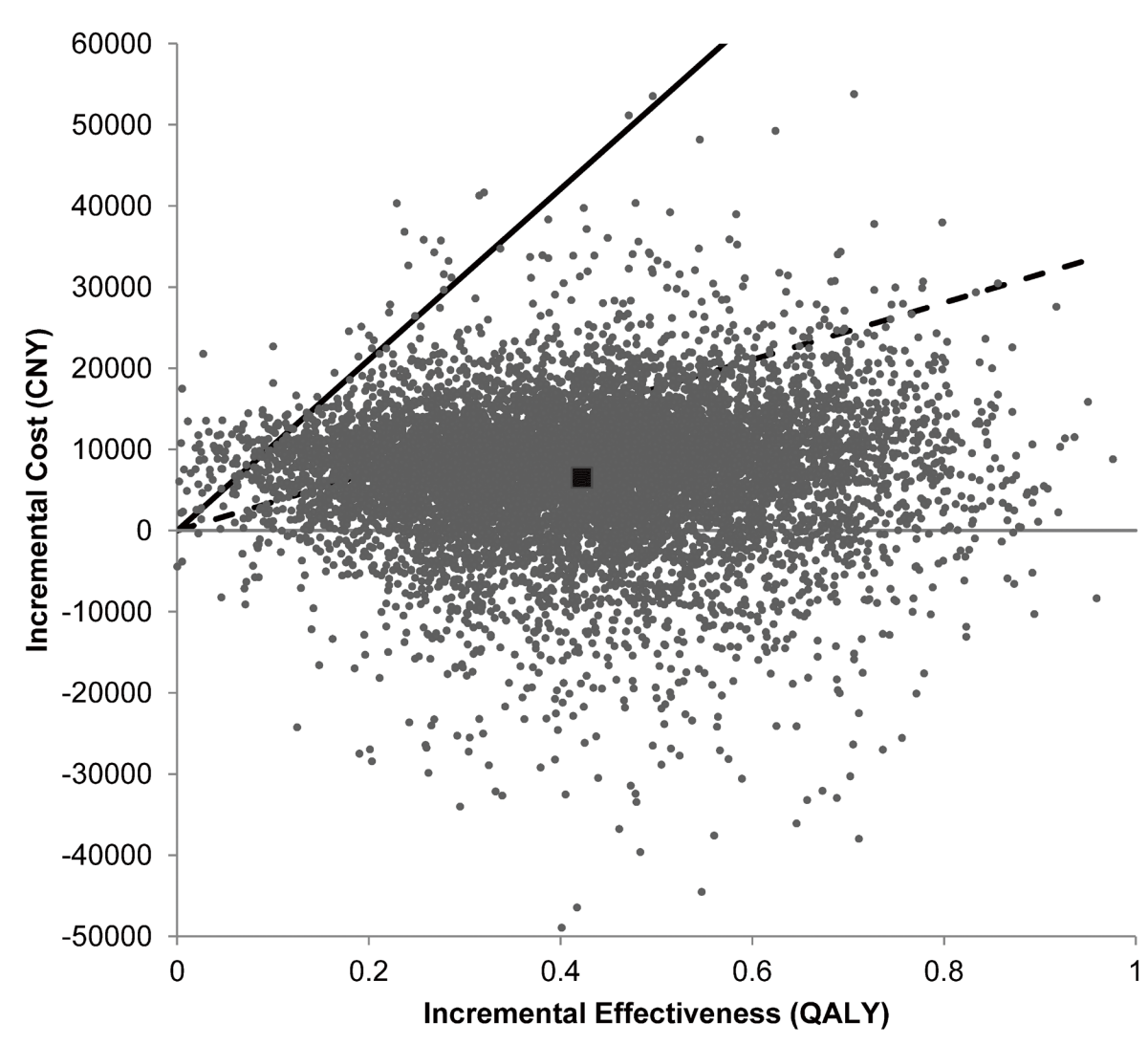

Figure 3. Scatterplot of the result of probabilistic sensitivity analysis comparing tPA treatment within 4.5 hours with no tPA treatment. Each point represents a simulation. The dark square represents the base case $(0.422$ QALYs gained at an incremental cost of CNY 6,530). The solid line represents the willingness-to-pay threshold of CNY 105,000 per QALY. The dashed line represents CNY 35,100 per QALY. Points to the right of the willingness-to-pay line are considered cost-effective.

doi:10.1371/journal.pone.0110525.g003 


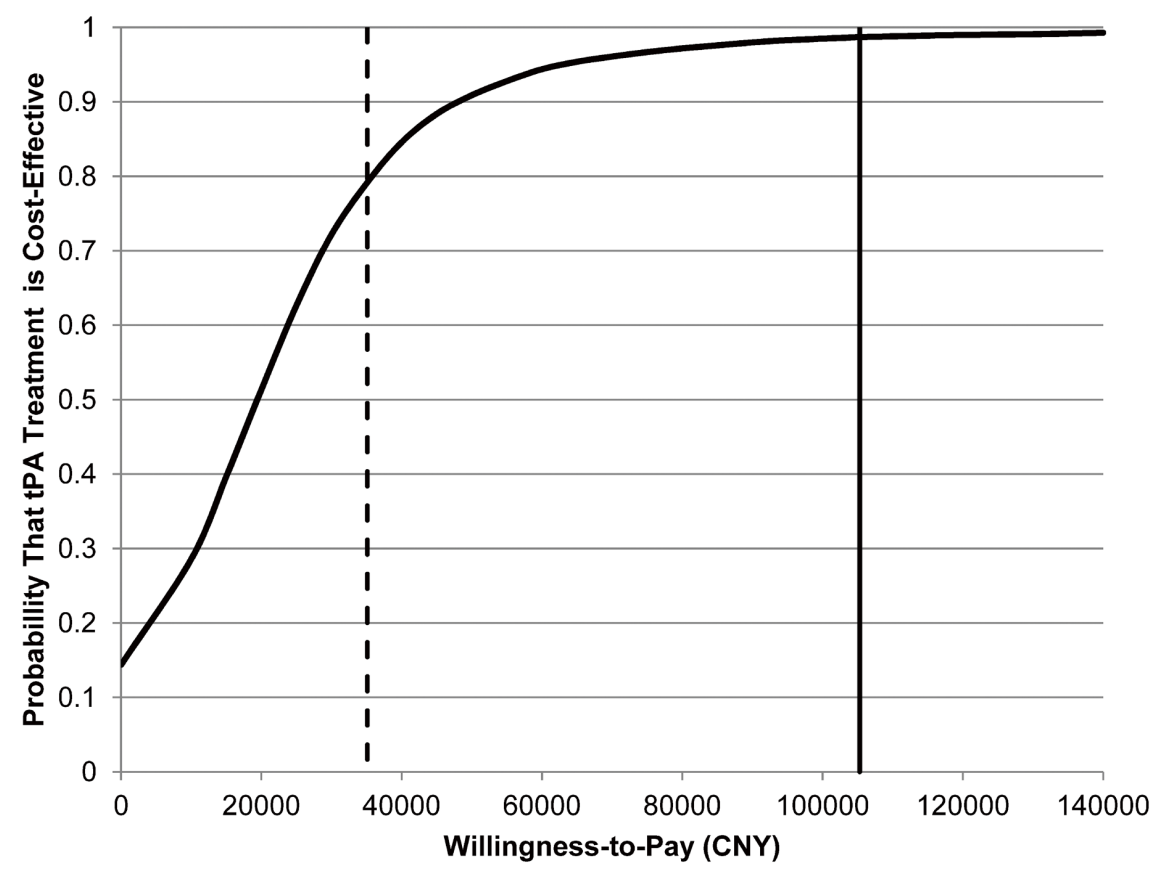

Figure 4. Cost-effectiveness acceptability curve. The curve presents the probability that tPA treatment within 4.5 hours to be cost-effective against willingness-to-pay threshold. The solid line represents the willingness-to-pay threshold of CNY 105,000 per QALY. The dashed line represents CNY 35,100 per QALY.

doi:10.1371/journal.pone.0110525.g004

costs of sICH applying the same rationale. Second, in China, the family plays an important role in both acute and post-acute care. This informal caregiving can be quite expensive. However, it was not considered in our model because of lack of corresponding data. Third, our model focused on the influence of tPA for acute ischemic stroke, and functional status and costs as a result of other causes, such as recurrence of intracranial hemorrhage, myocardial infarction, congestive heart failure, were not included in this model. In addition, the improved functional status after rehabilitation or other improvements was not considered in the model for lack of organized rehabilitation in China and lack of authentic data available on efficacy of rehabilitation or other improvements. Fourth, the efficacy of tPA treatment was based on the pooled analyses from the studies in developed countries (ECASS, ATLANTIS, NINDS, and EPITHET). Also, the utilities references were from Western literatures. We applied the efficacy of tPA treatment and the utilities to the model of Chinese patients, but did not know if these data fit Chinese patients. However, we ran sensitivity analysis and found that the results were robust for these parameters across plausible ranges. Although these limitations would have led to under- or over- estimation of the true costeffectiveness of tPA treatment in China, it is unlikely to make a significant differential impact on the overall results of our study because our findings were robust as shown in the sensitivity analyses.

\section{Conclusions}

The intravenous tPA within 4.5 hours for patients with acute ischemic stroke is highly cost-effective in China. Our study provides with constructive information on medical resource allocation for stroke treatment in China in future, and may also be a reference to other developing countries.

\section{Acknowledgments}

Special thanks to the physicians as well as all other personnel who dedicated themselves to the TIMS-CHINA project. The TIMS-CHINA investigators include: Yongjun Wang (Principle investigator, E-mail: ; yongjunwang1962@gmail.com), Beijing Tiantan Hospital; Xingquan Zhao, Beijing Tiantan Hospital; Yilong Wang, Beijing Tiantan Hospital; Xiaoling Liao, Beijing Tiantan Hospital; Chunxue Wang, Beijing Tiantan Hospital; Liping Liu, Beijing Tiantan Hospital; Chunjuan Wang, Beijing Tiantan Hospital; Yuesong Pan, Beijing Tiantan Hospital; Qi Bi, Beijing Anzhen Hospital; Liying Cui, Peking Union Medical College Hospital of Peking University; Yuheng Sun, Beijing Jishuitan Hospital; Maolin He, Beijing Shijitan Hospital; Dongsheng Fan, Peking University Third Hospital; Xiaojun Zhang, Beijing Tongren Hospital; Yansheng Li, Shanghai Renji Hospital; Shaoshi Wang, Shanghai First Municipal People's Branch hospital; Wei Fan, Zhongshan Hospital of Fudan University; Chuancheng Ren, The Fifth People's Hodpital of Shanghai, Fudan University; Zhenguo Liu, Xin Hua Hospital Affiliated to Shanghai Jiao Tong University; Xiaojiang Sun, The sixth People's Hospital Affiliated to Shanghai JiaoTong University; Xu Chen, Shanghai 8th People's Hospital; Qingke Bai, Pudong New Area People's Hospital; Dexiang Gu, Shanghai Yangpu Area Shidong Hospital; Chunmei $\mathrm{Hu}$, Shanghai Baoshan Area Center Hospital; Xin Li, Shanghai Yangpu Area Center Hospital; Qiang Dong, Huashan Hospital of Fudan University; Yan Cheng, Tianjin Medical University Gengeal Hospital; Bin Li, Dagang Oilfield Gengeal Hospital; Chen Li,Tianjin Fifth Center Hospital; Tongyu Wang, Bohai Oilfield Hospital; Liping Wang,Ninghe County People's Hospital of Tianjin; Kun Zhao, Baodi District People's Hospital of Tianjin; Dingbo Tao, The First Afflicated Hospital of Dlian Medical University; Fang Qu, Dalian Second People's hospital; Jingbo Zhang, Dalian Third People's hospital; Jianfeng Wang, Dalian Central hospital; Ying Lian, Dalian Economic and Technological Development District Hospital; Fang $\mathrm{Qu}$, Shenying Military District General hospital of Chinese People's Liberation Army; Ying Gao, National Traditional Chinese Medicine (TCM)Thrombus Treatment Center of Liaoning Province; Huashan Sun, Jilin Chemical Industrial Group General hopital; Jinying Li, Jilin Oilfield General Hospital; Guozhong Li, The First Clinical College of Harbin Medical University; Yulan Zhu, The Second Clinical College of Harbin Medical University; Zichao Yang, The Fourth Clinical College of Harbin 
Medical University; Jun Zhou, Mudan Jiang Second hospital; Minxia Guo, Shaanxi Provincial People's Hospital; Qilin Ma, The First Hospital of Xiamen; Xiaoping Gao, Hunan Provincial People's Hospital; Renbin Huang, Chenzhou First People's Hospital; Bo Xiao, Xiangya Hospital of Centre-south University; Kangning Chen, Southwest Hospital; Li Gao, Chengdu Third Municipal People's Hospital; Anding Xu, The First Affiliated Hospital of Jinan University; Ming Shao, The First Affiliated Hospital of Guangzhou Medical University; En Xu, The Second Affiliated Hospital of Guangzhou Medical University; Xiaoping Pan, Guangzhou First Municipal People's Hospital; Yefeng Cai, Guangdong Provincial Hospital of Traditional Chinese Medicine; Yun Xu, Drum Tower Hospital Affiliated to Nanjing Medical University; KaiFu Ke, The Affiliated Hospital of Nantong University; Yuenan Kong,Wuxi Second People's Hospital; Liangcai Ding, The Third Affiliated Hospital of Suzhou University; Yibin Cao, Tangshan Gongren Hospital; Yumin Liu, Zhongnan Hospital of Wuhan University; Kang Xu, Hubei Xinhua Hospital; Chengming Xing, Qingdao Municipal Hospital Group; Shaohua

\section{References}

1. Yang G, Wang Y, Zeng Y, Gao GF, Liang X, et al. (2013) Rapid health transition in China, 1990-2010: findings from the Global Burden of Disease Study 2010. Lancet 381: 1987-2015.

2. Hacke W, Kaste M, Fieschi C, Toni D, Lesaffre E, et al. (1995) Intravenous thrombolysis with recombinant tissue plasminogen activator for acute hemispheric stroke. The European Cooperative Acute Stroke Study (ECASS). JAMA 274: $1017-1025$.

3. The National Institute of Neurological Disorders and Stroke rt-PA Stroke Study Group (1995) Tissue plasminogen activator for acute ischemic stroke. N Engl J Med 333: 1581-1587.

4. Hacke W, Donnan G, Fieschi C, Kaste M, von Kummer R, et al. (2004) Association of outcome with early stroke treatment: pooled analysis of ATLANTIS, ECASS, and NINDS rt-PA stroke trials. Lancet 363: 768-774.

5. Hacke W, Kaste M, Bluhmki E, Brozman M, Dávalos A, et al. (2008) Thrombolysis with alteplase 3 to 4.5 hours after acute ischemic stroke. N Engl J Med 359: 1317-1329.

6. Wahlgren N, Ahmed N, Dávalos A, Hacke W, Millán M, et al. (2008) Thrombolysis with alteplase $3-4.5 \mathrm{~h}$ after acute ischaemic stroke (SITS-ISTR): an observational study. Lancet 372: 1303-1309.

7. Fagan SC, Morgenstern LB, Petitta A, Ward RE, Tilley BC, et al. (1998) Costeffectiveness of tissue plasminogen activator for acute ischemic stroke. NINDS rt-PA Stroke Study Group. Neurology 50: 883-890.

8. Sinclair SE, Frighetto L, Loewen PS, Sunderji R, Teal P, et al. (2001) CostUtility analysis of tissue plasminogen activator therapy for acute ischaemic stroke: a Canadian healthcare perspective. Pharmacoeconomics 19: 927-936.

9. Sandercock P, Berge E, Dennis M, Forbes J, Hand P, et al. (2004) Costeffectiveness of thrombolysis with recombinant tissue plasminogen activator for acute ischemic stroke assessed by a model based on UK NHS costs. Stroke 35: 1490-1497.

10. Ehlers L, Andersen G, Clausen LB, Bech M, Kjølby M (2007) Cost-effectiveness of intravenous thrombolysis with alteplase within a 3-hour window after acute ischemic stroke. Stroke 38: 85-89.

11. Jung KT, Shin DW, Lee KJ, Oh M (2010) Cost-effectiveness of recombinant tissue plasminogen activator in the management of acute ischemic stroke: a systematic review. J Clin Neurol 6: 117-126.

12. Tung CE, Win SS, Lansberg MG (2011) Cost-effectiveness of tissue-type plasminogen activator in the 3 - to 4.5 -hour time window for acute ischemic stroke. Stroke 42: 2257-2262.

13. Kim AS, Nguyen-Huynh M, Johnston SG (2011) A cost-utility analysis of mechanical thrombectomy as an adjunct to intravenous tissue-type plasminogen activator for acute large-vessel ischemic stroke. Stroke 42: 2013-2018.

14. Boudreau DM, Guzauskas G, Villa KF, Fagan SC, Veenstra DL (2012) A model of cost-effectiveness of tissue plasminogen activator in patient subgroups 3 to 4.5 hours after onset of acute ischemic stroke. Ann Emerg Med 61: 46-55.

15. Tan Tanny SP, Busija L, Liew D, Teo S, Davis SM, et al. (2013) Costeffectiveness of thrombolysis within 4.5 hours of acute ischemic stroke: experience from Australian stroke center. Stroke 44: 2269-2274.

16. Kim AS, Johnston SC (2013) Temporal and geographic trends in the global stroke epidemic. Stroke 44: S123-S125.

17. Wang Y, Liao X, Zhao X, Wang DZ, Wang C, et al. (2011) Using recombinant tissue plasminogen activator to treat acute ischemic stroke in China: analysis of the results from the Chinese National Stroke Registry (CNSR). Stroke 42: 16581664 .

18. Demaerschalk BM, Durocher DL (2007) How diagnosis-related group 559 will change the US Medicare cost reimbursement ratio for stroke centers. Stroke 38: $1309-1312$.
Su, Dezhou People's Hospital; Guiru Zhang, Penglai People's Hospital; Rongyuan Zheng, The First Affiliated Hospital of Wenzhou Medical University; Ming Zhai, First People's Hospital of Yunnan Province; Yi Zhu, The Xinjiang Autonomous Region People's Hospital; Yuanxin Liu, Autonomous Region TCM Hospital Affiliated to Xinjiang Medical University; Xiaoying Zhang, Hospital of Xinjiang Production \& Construction Corps; Shizheng Wu, Qinghai Provincial People's Hospital; Jinfeng Liu, Yangquan Coalmine Group General hospital. In addition, we thank Dr. Jiming Fang for helpful edits on the manuscript.

\section{Author Contributions}

Conceived and designed the experiments: YSP Yilong Wang Yongjun Wang. Performed the experiments: QDC XQZ XLL Chunjuan Wang. Analyzed the data: YSP GFL Yilong Wang. Contributed reagents/ materials/analysis tools: XQZ WLD LPL. Wrote the paper: YSP Chunxue Wang Yilong Wang Yongjun Wang.

19. Peltola M, Quentin W (2013) Diagnosis-related groups for stroke in Europe: patient classification and hospital reimbursement in 11 countries. Cerebrovasc Dis 35: 113-123.

20. Beijing Municipal Commission of Development and Reform. The maximum retail price of western medicine (in Chinese). Website: http://service2.bjpc.gov. cn/bjpc/mediprice/WesternMedicine_qry.jsp. Accessed 2013 July 28.

21. Liao XL, Wang CX, Wang YL, Wang CJ, Zhao XQ et al. (2012) Thrombolysis Implementation and Monitor of acute ischemic Stroke in China (TIMS-China) Investigators. Implementation and outcome of thrombolysis with alteplase 3 to $4.5 \mathrm{~h}$ after acute stroke in Chinese patients. CNS Neurosci Ther 19: 43-47.

22. Weinstein MC, Siegel JE, Gold MR, Kamlet MS, Russell LB (1996) Recommendations of the Panel on Cost-effectiveness in Health and Medicine. JAMA 276: 1253-1258.

23. Lees KR, Bluhmki E, von Kummer R, Brott TG, Toni D, et al. (2010) Time to treatment with intravenous alteplase and outcome in stroke: an updated pooled analysis of ECASS, ATLANTIS, NINDS, and EPITHET trials. Lancet 375: 1695-1703.

24. Wang Y, Cui L, Ji X, Dong Q Zeng J, et al. (2011) The China National Stroke Registry for patients with acute cerebrovascular events: design, rationale, and baseline patient characteristics. Int J Stroke 6: 355-361.

25. National Bureau of Statistics of China. The 2010 Population Census of the Peaple's Republic of China (in Chinese). Website: http://www.stats.gov.cn/tjsj/ pcsj/rkpc/6rp/indexch.Htm. Accessed 2012 October 25.

26. Ministry of Health of the People's Republic of China (2012) China Health Statistics Yearbook 2012. Beijing: Peking Union Medical College Press (in Chinese).

27. Samsa GP, Reutter RA, Parmigiani G, Ancukiewicz M, Abrahamse P, et al. (1999) Performing cost-effectiveness analysis by integrating randomized trial data with a comprehensive decision model: application to treatment of acute ischemic stroke. J Clin Epidemiol 52: 259-271.

28. Hylek EM, Go AS, Chang Y, Jensvold NG, Henault LE, et al. (2003) Effect of intensity of oral anticoagulation on stroke severity and mortality in atrial fibrillation. N Engl J Med 349: 1019-1026.

29. Lee S, Anglade MW, Pham D, Pisacane R, Kluger J, et al. (2012) Costeffectiveness of rivaroxaban compared to warfarin for stroke prevention in atrial fibrillation. Am J Cardiol 110: 845-851.

30. You JH, Tsui KK, Wong RS, Cheng G (2012) Cost-effectiveness of dabigatran versus genotype-guided management of warfarin therapy for stroke prevention in patients with atrial fibrillation. PLoS One 7: e39640.

31. Commission on Macroeconomics and Health of World Health Organization. CHOosing Interventions that are Cost Effective (WHO-CHOICE). Websit: http://www.who.int/choice/costs/CER_thresholds/en/. Accessed 2012 October 17 .

32. Heeley E, Anderson CS, Huang Y, Jan S, Li Y, et al. (2009) Role of health insurance in averting economic hardship in families after acute stroke in China. Stroke 40: 2149-2156.

33. Li A, Huang ZQ, Liu HT, Deng YT, Xiao JW, et al. (2009) Knowledge, attitude and behavior for stroke and rehabilitation in stroke patients and relatives. (in Chinese). Chin J Rehabil Theory and Pract 15: 252-254.

34. Gao YL, Chen LD, Tao J, Chen SM (2009) Investigation on the investment and popularization of rehabilitation from cerebral apoplexy: in 2 communities of Xiamen City. (in Chinese). Chin J Convalescent Med 18: 472-474.

35. Ma R, Wang C, Zhao X, Xu M, Lv Y, et al. (2008) A survey on compliance with secondary stroke prevention guidelines and follow up for the inpatients with atherosclerotic cerebral infarction/transient ischemic attack. Neurol Res 30: 383-388.

36. Wei JW, Wang JG, Huang Y, Liu M, Wu Y, et al. (2010) Secondary prevention of ischemic stroke in urban China. Stroke 41: 967-974. 